\title{
Distribución de Tetrasporidium javanicum Möbius, 1893 (Chlorophyta: Tetrasporales) en la Cuenca del río Ebro
}

\author{
Pedro Tomás ${ }^{1, *}$, José Luis Moreno ${ }^{2}$, Marina Aboal $^{3}$, Javier Oscoz ${ }^{4}$, Concha Durán ${ }^{5}$ y Patricia \\ Navarro $^{5}$ \\ ${ }^{1}$ Laboratorio de Ensayos Técnicos S.A. ENSAYA. Pol. Ind. Valdeconsejo C/Aneto parcela 8-A ,50410 Cuarte de \\ Huerva (Zaragoza). Tlf. 976566875, Fax. 976566612. \\ 2 Centro Regional de Estudios del Agua (CREA), Universidad de Castilla-La Mancha, Ctra. de las Peñas, km 3, \\ 020701 Albacete. \\ ${ }^{3}$ Departamento de Biología Vegetal. Facultad de Biología. Universidad de Murcia. Campus de Espinardo, \\ 30100, Espinardo. Murcia. \\ ${ }^{4}$ Departamento de Zoología y Ecología, Universidad de Navarra, Apdo. 177, 31080 Pamplona (Navarra). \\ 5 Área de Calidad de Aguas, Confederación Hidrográfica del Ebro, Paseo de Sagasta 24-28, 50071 Zaragoza. \\ * Autor responsable de la correspondencia: biologia@ensaya.es
}

Recibido: $17 / 11 / 2010$

Aceptado: 8/7/2011

\begin{abstract}
Distribution of Tetrasporidium javanicum Möbius, 1893 (Chlorophyta: Tetrasporales,) in the Ebro River Basin

During the implementation of the biological samplings in the CEMAS network (Control of the Status of Surface Water Bodies) in the Ebro River Basin, in application of the Water Framework Directive (period 2006-2010), the presence of the chlorophycean alga Tetrasporidium javanicum Möbius, 1893 was detected in the middle reaches of Ebro River, in lowland reaches of the main rivers of the left margin, and in some upper reaches, gullies and also in irrigation ditches. It is a species that is distributed mainly in tropical areas that until now had been scarcely reported in Spain and Europe. In this work, the distribution and ecological preferences of the species in the River Ebro Basin is shown.
\end{abstract}

Key words: Green algae, macrophytes, rivers, Spain.

\section{RESUMEN}

Distribución de Tetrasporidium javanicum Möbius, 1893 (Chlorophyta: Tetrasporales) en la cuenca del río Ebro

Durante la realización de los muestreos biológicos de la red CEMAS (Control del Estado de las Masas de Agua Superficiales) de la Cuenca del Ebro en aplicación de la Directiva Marco del Agua (periodo 2006-2010), se detectó la presencia del alga clorofícea Tetrasporidium javanicum Möbius, 1893 en el tramo medio del río Ebro, en los tramos bajos de los principales ríos de la margen izquierda, así como en algún tramo alto, barrancos y acequias de riego. Se trata de una especie principalmente de zonas tropicales que hasta ahora había sido escasamente citada en España y Europa. En este trabajo se presenta la distribución y preferencias ecologicas de la especie en la Cuenca del Ebro.

Palabras clave: Algas verdes, macrófitos, ríos, España.

\section{INTRODUCCIÓN}

La aplicación de la Directiva Marco del Agua (DMA) (Directiva 2000/60/CE), establece la necesidad de estudiar los diferentes componentes bióticos de nuestros ríos: macrófitos, diatomeas, macroinvertebrados, peces y la vegetación de ribera. La aplicación de esta Directiva está suponiendo el estudio profundo de nuestros ríos de forma estandarizada en todo el territorio penin- 
sular. Como consecuencia de este muestreo en amplias redes de control de calidad, se está generando un aumento en el conocimiento de la biodiversidad de nuestra flora acuática. Cabe destacar la presencia de Tetrasporidium javanicum, un clorófito del orden Tetrasporales, abundante en zonas tropicales de la India (Iyengar, 1932), China (Hu \& Wei, 2006), Java, Estados Unidos, Australia y Bangladesh (Entwisle \& Skinner, 2000), aunque también ha sido recolectada en diversas localidades del continente europeo: República Checa (Fott, 1965), Francia (Coute \& Tracanna, 1981) y Portugal (Calado \& Rino, 1992). En España se ha citado en arroyos del sureste peninsular y canales de riego de Extremadura (Aboal et. al, 1994, 2006; Marín-Murcia \& Aboal, 2007) y en arroyos silíceos del sur y norte de España (Moreno et al., 2011; Penalta-Rodríguez \& López-Rodríguez, 2007).

En el presente estudio se presenta el estado de conocimiento sobre la distribución actual de Tetrasporidium javanicum en la Cuenca del Ebro, de acuerdo a los datos recolectados en los últimos años, describiendo las condiciones ecológicas de las aguas en las que se ha hallado.

\section{METODOLOGÍA}

Durante los meses de Junio a Septiembre en el periodo 2006-2010, se visitaron entre 211 (2006) y 362 (2009) estaciones de muestreo pertenecientes a las redes de control operativo, vigilancia y referencia definidas en las masas de agua de la Cuenca del Ebro. En total se tomaron y analizaron 1200 muestras de macrófitos. En cada una de las estaciones, se seleccionaron tramos representativos de unos $100 \mathrm{~m}$ de longitud, donde se realizaron recorridos en zig-zag y se recolectaron todos los macrófitos visibles a simple vista, ya sea a mano o con una espátula-cuchara si se encontraban fuertemente adheridos al sustrato. Posteriormente, se conservaron convenientemente etiquetados con formol al $4 \%$ y se llevaron al laboratorio para proceder a su determinación taxonómica con lupa binocular y microscopio (CHE, 2005). En el campo se midieron diferentes parámetros físico-químicos del agua $\left(\mathrm{pH}, \mathrm{T}^{\mathrm{a}}\right.$, conductividad y oxígeno disuelto) mediante una sonda multiparamétrica YSI 556 MPS, además de tomar muestras de agua para analizar en el laboratorio la concentración de nitratos, nitritos, amonio y fosfatos (fósforo reactivo soluble). Los análisis de agua se realizaron según las indicaciones de la ITC-MMA.EECC-1/06 (MMA, 2006). Una vez realizado el muestreo de cada una de las estaciones, se procedió a la desinfección del material y de los equipos de muestreo utilizados de acuerdo al protocolo establecido por la CHE (2007) para evitar la propagación del mejillón cebra y otros organismos nocivos en nuestros ríos. Para la realización del mapa de distribución y la evaluación de los parámetros ecológicos se utilizaron los datos de las redes de control biológico de los años 2006, 2007, 2008, 2009 y 2010 que se encontraban disponibles, así como observaciones esporádicas personales.

Las observaciones microscópicas y las fotografías se realizaron con un microscopio Olympus BX50 equipado con un set Colorview II. Una parte del material se prensó y se conservó en pliegos de herbario que se depositaron en el herbario de la Universidad de Murcia (MUB).

\section{RESULTADOS}

En el río Ebro, Tetrasporidium javanicum desarrolla colonias fijadas al sustrato, mucilaginosas, globosas primero y laminares con perforaciones en el estado maduro (Fig. 1:1). Estas colonias pueden medir desde menos de $1 \mathrm{~cm}$ hasta los $15 \mathrm{~cm}$ de longitud y están claramente clatradas (Fig. 1: 2-3). Las células suelen distribuirse de forma homogénea en el mucílago colonial, aunque en las diferentes fases de la división celular puede observarse agrupadas por parejas o en tétradas (Fig. 1: 4-6). Las células son subesféricas a elipsoidales $(6-11 \mu \mathrm{m}$ de longitud) y poseen cloroplasto parietal de color verde intenso con un gran pirenoide (de 1-3 $\mu \mathrm{m}$ ). En ningún caso se observaron los pseudoflagelos que caracterizan al género Tetraspora. En los especímenes vivos las vacuolas pulsátiles apicales son muy visibles.

La distribución de T. javanicum en los ríos de la Cuenca del Ebro se circunscribe al eje 
principal del río Ebro y a los tramos medios y bajos de sus principales afluentes de la mar- gen izquierda, así como a acequias y barrancos en torno al eje fluvial principal (Fig. 2). En
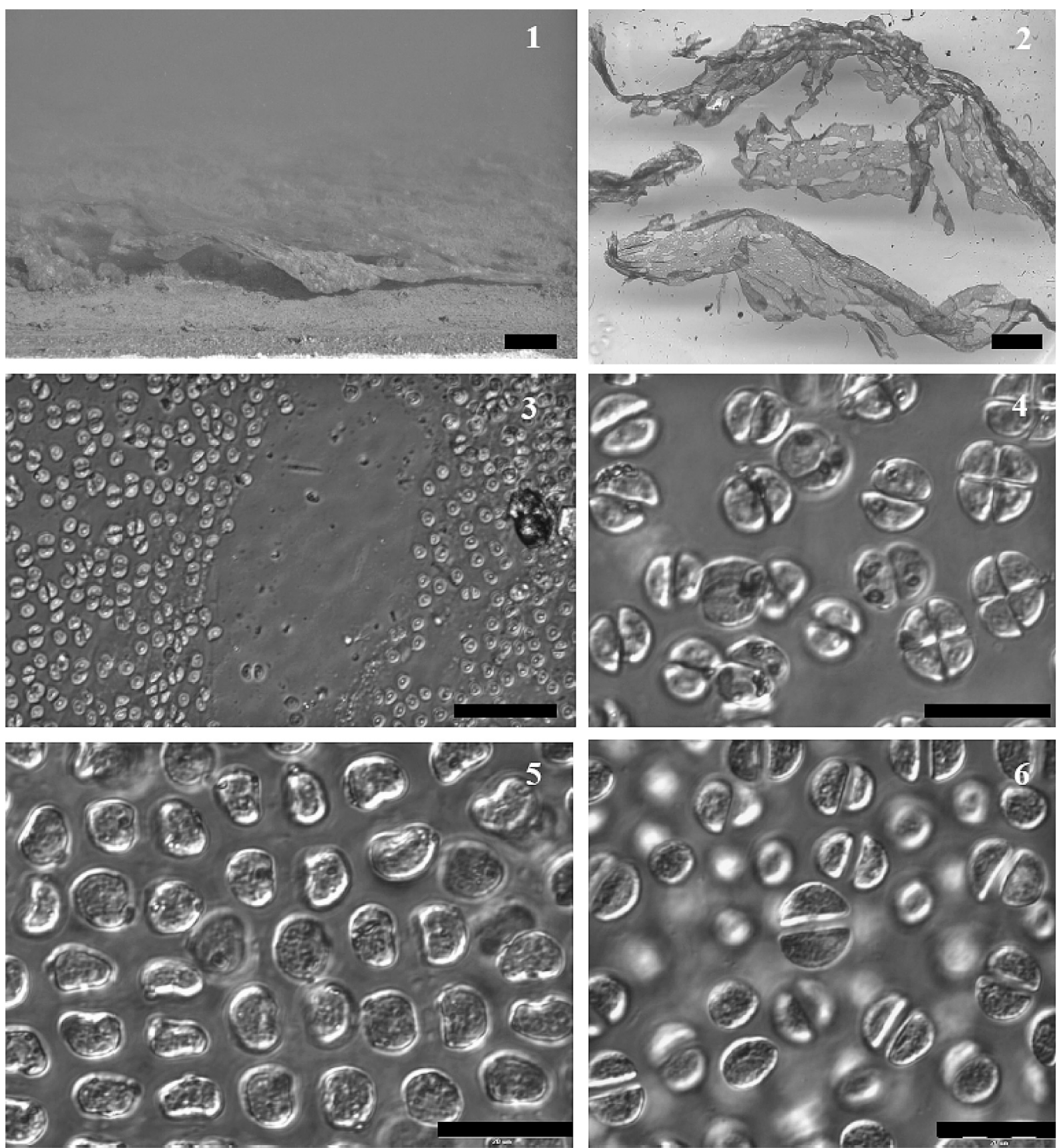

Figura 1. 1) Aspecto del talo en un canal de riego. 2) Detalle del talo clatrado. 3) Detalle de un hiato de un espécimen del río Aragón. 4) Células en división en talos de una acequia de Sariñena. 5) Disposición de las células en un talo maduro del río Cinca. 6) Células en diferentes fases de división en material del Puente de Elciego. La escala representa $1 \mathrm{~cm}$ en $1-2,50 \mu \mathrm{m}$ en 3 y $20 \mu \mathrm{m}$ en 4-6. 1) Thallus appearance in an irrigation channel. 2) Detailed view of the clathrate thallus. 3) Detail of a hiatus in a specimen of the Aragón River. 4) Cell division in thallus from a Sarinena ditch. 5) Cells disposition in a mature thallus from the Cinca Rriver. 6) Cells in different phases of cell division in samples from Puente de Elciego. The scale represents $1 \mathrm{~cm}$ in $1-2,50 \mu \mathrm{m}$ in 3 and $20 \mu \mathrm{m}$ in $4-6$. 


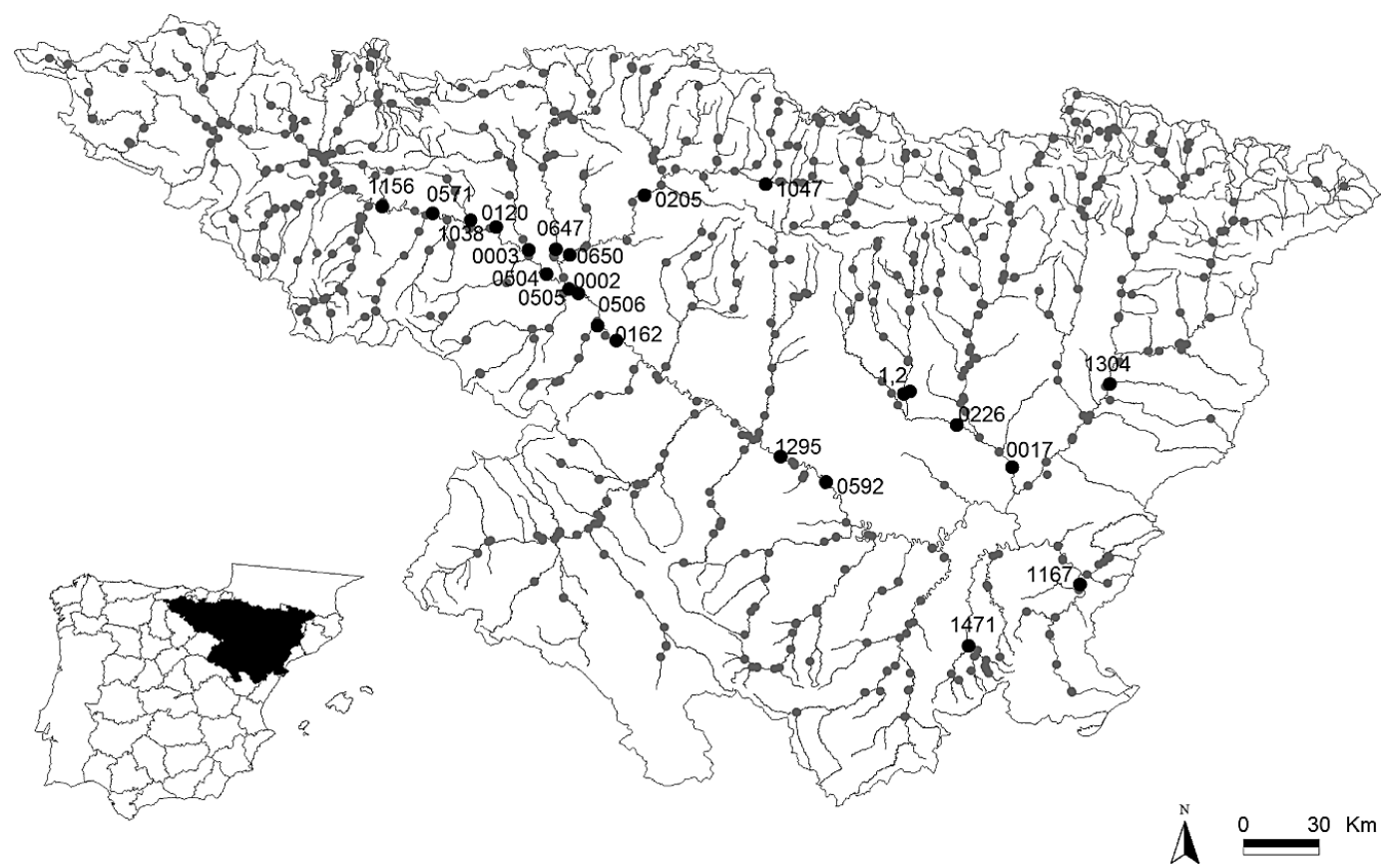

Figura 2. Distribución de T. javanicum en la Cuenca del Ebro. Puntos negros: presencia; Puntos grises: ausencia. Distribution of T. javanicum in the Ebro River Basin. Black dots: presence; grey dots: absence.

total, se ha encontrado en 23 tramos fluviales pertenecientes a cinco de las tipologías oficiales definidas por el Centro de Estudios y Experimentación de Obras Públicas (CEDEX): 115 (Ejes mediterráneo-continentales poco mineralizados), 117 (Grandes ejes en ambiente mediterráneo), 109 (Ríos mineralizados de baja montaña mediterránea), 112 (Ríos de montaña mediterránea calcárea) y 126 (Ríos de montaña húmeda calcárea) (Tabla 1 y Fig. 2). Se trata de tramos de la cuenca entre 19 y $600 \mathrm{~m}$ de altitud, localizados en una zona eminentemente agrícola donde predomina la agricultura intensiva de regadío.

La especie se ha recolectado principalmente en zonas de orilla con una velocidad del flujo del agua baja o nula, aunque también se observó en las orillas de rápidos poco profundos flotando, quedando casi por completo emergida. El sustrato del cauce estaba dominado por cantos y gravas, aunque también se observó sobre limo y tallos de Phragmites australis (Cav.) Trin. ex Steudel. Tetrasporidium javanicum se halló junto a otros clorófitos (Cladophora sp., Enteromorpha sp., Spirogyra sp., Oedogonium sp., Stigeoclo- nium sp.), cianofíceas (Oscillatoria sp., Phormidium sp., Nostoc sp.), rodofíceas (Thorea hispida (Thore) Desvaux, Compsopogon coeruleus (Balbis) Montagne, Hildenbrandia rivularis (Liebmann) J.Agardh, Bangia atropurpurea (A. Roth) C. Agardh) y algunas plantas vasculares (Myryophyllum sp., Potamogeton sp. y Ranunculus sp.).

La mayor parte de los especímenes se recolectaron en aguas turbias, de temperatura elevada (16.2-26.6 $\left.{ }^{\circ} \mathrm{C}\right)$, básicas ( $\left.\mathrm{pH} 7.6-8.4\right)$, de conductividad intermedia (481-3581 $\mu \mathrm{S} / \mathrm{cm})$ y con una concentración de oxígeno disuelto variable, oscilando entre 5.21 y $10.40 \mathrm{mg} / 1 \mathrm{O}_{2}$ (Tabla 2). Sin embargo también se han recolectado ejemplares en las estaciones 0205, 1047 y 1471, de aguas transparentes, temperatura elevada $\left(16.20-24.9{ }^{\circ} \mathrm{C}\right)$, conductividad mediabaja $(245-490 \mu \mathrm{S} / \mathrm{cm})$ y bien oxigenadas (8.35$11.77 \mathrm{mg} / \mathrm{l}_{2}$ ). El rango trófico observado es amplio, llegando a alcanzar concentraciones altas de nitrato $\left(6.38-20.1 \mathrm{mg} / \mathrm{NO}_{3}\right)$, amonio $(<0.05$ $\left.0.32 \mathrm{mg} / \mathrm{NH}_{4}\right)$ y ortofosfato $(<0.03-0.52 \mathrm{mg} / \mathrm{l}$ $\mathrm{PO}_{4}$ ). Por tanto, de forma global se puede afirmar que, en la cuenca del Ebro, se trata de una 
Tabla 1. Localidades en las que se encontró T. javanicum. Tipología: 117: Grandes ejes en ambiente mediterráneo; 115: Ejes mediterráneo-continentales poco mineralizados; 109: Ríos mineralizados de baja montaña mediterránea; 112: Ríos de montaña mediterránea calcárea; 126: Ríos de montaña húmeda calcárea. Sites where T. javanicum was found. Typology: 117: Large axes in a mediterranean environment; 115: Poorly mineralised continental-mediterranean axes; 109: Mineralised low mountain mediterranean rivers; 112: Limestone mediterranean mountain rivers, 126: Wet limestone mountain rivers.

\begin{tabular}{|c|c|c|c|c|c|c|}
\hline Código & Toponimia & Fecha & Tipología & Altitud (msnm) & UTM X & UTM Y \\
\hline 0002 & Ebro / Castejón & $10 / 08 / 2008$ & 117 & 260 & 608539 & 4670730 \\
\hline \multirow[t]{2}{*}{0003} & Ega / San Adrián & 09/07/2009 & 115 & 298 & 588737 & 4687874 \\
\hline & & $29 / 07 / 2010$ & & & & \\
\hline \multirow[t]{4}{*}{0017} & Cinca / Fraga & $02 / 08 / 2007$ & 115 & 91 & 779357 & 4602264 \\
\hline & & $21 / 07 / 2008$ & & & & \\
\hline & & $03 / 08 / 2009$ & & & & \\
\hline & & $12 / 08 / 2010$ & & & & \\
\hline 0120 & Ebro / Mendavia (Der. Canal Lodosa) & 09/07/2009 & 115 & 317 & 576031 & 4696947 \\
\hline \multirow[t]{2}{*}{0162} & Ebro / Pignatelli & 06/07/2009 & 117 & 251 & 623341 & 4652117 \\
\hline & & $07 / 09 / 2010$ & & & & \\
\hline 0205 & Aragón / Cáseda & $20 / 09 / 2010$ & 115 & 378 & 634415 & 4709625 \\
\hline 0226 & Alcanadre / Ontiñena & $09 / 08 / 2010$ & 109 & 159 & 757442 & 4618951 \\
\hline 0504 & Ebro / Rincón de Soto & $14 / 09 / 2010$ & 115 & 280 & 596000 & 4678425 \\
\hline 0505 & Ebro / Alfaro & 07/07/2009 & 117 & 265 & 604750 & 4672390 \\
\hline \multirow[t]{2}{*}{0506} & Ebro / Tudela & 06/07/2009 & 117 & 253 & 615942 & 4658112 \\
\hline & & $07 / 09 / 2010$ & & & & \\
\hline \multirow[t]{3}{*}{0571} & Ebro / Logroño - Varea & $13 / 08 / 2008$ & 115 & 357 & 551112 & 4702431 \\
\hline & & $13 / 07 / 2009$ & & & & \\
\hline & & $14 / 09 / 2010$ & & & & \\
\hline 0592 & Ebro / Pina de Ebro & $08 / 09 / 2010$ & 117 & 160 & 705956 & 4596466 \\
\hline \multirow[t]{2}{*}{0647} & Arga / Peralta & $28 / 06 / 2007$ & 115 & 291 & 599680 & 4688268 \\
\hline & & 08/07/2009 & & & & \\
\hline 0650 & Aragón / Derivación Acequia Río Molinar & $28 / 06 / 2007$ & 115 & 294 & 605089 & 4685944 \\
\hline 1038 & Linares / Mendavia & $29 / 07 / 2010$ & 109 & 350 & 566023 & 4699735 \\
\hline 1047 & Aragón / Puente la Reina de Jaca & $18 / 08 / 2010$ & 126 & 600 & 681997 & 4713948 \\
\hline \multirow[t]{3}{*}{1156} & Ebro / Puente de Elciego & $19 / 08 / 2008$ & 115 & 400 & 531049 & 4705077 \\
\hline & & $15 / 07 / 2009$ & & & & \\
\hline & & $14 / 09 / 2010$ & & & & \\
\hline 1167 & Ebro / Mora de Ebro & $15 / 07 / 2008$ & 117 & 19 & 806108 & 4555809 \\
\hline 1295 & Ebro / El Burgo de Ebro & $02 / 07 / 2009$ & 117 & 179 & 687957 & 4606508 \\
\hline 1304 & Sio / Balaguer E.A. 182 & $03 / 08 / 2008$ & 109 & 236 & 817929 & 4635212 \\
\hline 1471 & Matarraña / Aguas arriba desembocadura del Tastavins & $01 / 07 / 2010$ & 112 & 457 & 762229 & 4531568 \\
\hline 1 & Acequia / Sariñena & $02 / 11 / 2009$ & 109 & 240 & 736636 & 4631200 \\
\hline 2 & Bco. Malfarás / Sariñena & $02 / 04 / 2010$ & 109 & 274 & 739062 & 4632121 \\
\hline
\end{tabular}

especie característica de aguas eutróficas cálidas en tramos medios y bajos, y que puede soportar concentraciones elevadas de sales disueltas.

\section{DISCUSIÓN}

Las características morfológicas de los ejemplares hallados en la cuenca del Ebro, coinciden con las típicas de la especie (Fott et al., 1965; Ettl \& Gardner, 1988). Esta especie es bien conocida en áreas tropicales de Australia, China, Bangladesh (Entwistle \& Skinner, 2000) y Estados Unidos (Islas Hawai) (Sherwood, 2004). En Europa se ha citado en Francia, en la República Checa y en Portugal pero siempre en sistemas acuáticos de aguas transparentes a turbias o de zonas agrícolas (Coute \& Tracanna, 1981; Calado \& 
Tabla 2. Parámetros físico-químicos de las localidades en las que se encontró T. javanicum en la Cuenca del Ebro. (<: valores inferiores al límite de cuantificación del método analítico). Physichochemical parameters of the locations where T. javanicum was found in the River Ebro Basin. (<: values below the quantification limit of the analytical method).

\begin{tabular}{|c|c|c|c|c|c|c|c|c|c|}
\hline Código & Fecha & Temperatura ${ }^{\circ} \mathrm{C}$ & pH & Conductividad $(\mu \mathrm{S} / \mathrm{cm})$ & $\mathrm{O}_{2}(\mathrm{mg} / \mathrm{l})$ & $\mathrm{NO}_{2}(\mathrm{mg} / \mathrm{l})$ & $\mathrm{NO}_{3}(\mathbf{m g} / \mathbf{l})$ & $\mathrm{NH}_{4}(\mathrm{mg} / \mathrm{l})$ & $\mathrm{PO}_{4}(\mathrm{mg} / \mathrm{l})$ \\
\hline 0002 & $10 / 08 / 2008$ & 19.53 & 7.56 & 862 & 9.03 & $<0.01$ & 7.20 & 0.05 & $<0.03$ \\
\hline 0003 & 09/07/2009 & 20.03 & 8.09 & 3215 & 8.20 & $<0.06$ & 14.52 & $<0.05$ & 0.21 \\
\hline 0003 & $29 / 07 / 2010$ & 22.72 & 8.01 & 3581 & 8.59 & & & & \\
\hline 0017 & $02 / 08 / 2007$ & 24.23 & 8.03 & 1180 & 7.04 & 0.15 & 9.74 & 0.30 & 0.21 \\
\hline 0017 & $21 / 07 / 2008$ & 22.18 & 8.17 & 975 & 9.27 & $<0.01$ & 7.57 & $<0.05$ & 0.19 \\
\hline 0017 & 03/08/2009 & 23.61 & 7.97 & 1434 & 7.40 & $<0.06$ & 14.47 & $<0.05$ & 0.08 \\
\hline 0017 & $12 / 08 / 2010$ & 22.94 & 8.09 & 1130 & 8.81 & & & & \\
\hline 0120 & 09/07/2009 & 22.21 & 7.94 & 784 & 8.04 & $<0.06$ & 8.70 & $<0.05$ & 0.43 \\
\hline 0162 & 06/07/2009 & 24.40 & 7.81 & 1190 & 8.59 & 0.33 & 10.90 & 0.08 & 0.29 \\
\hline 0162 & $07 / 09 / 2010$ & 21.49 & 7.85 & 1157 & 8.38 & & & & \\
\hline 0205 & $20 / 09 / 2010$ & 16.20 & 7.96 & 245 & 10.28 & & & & \\
\hline 0226 & $09 / 08 / 2010$ & 24.63 & 8.19 & 1052 & 9.39 & & & & \\
\hline 0504 & $14 / 09 / 2010$ & 20.91 & 7.90 & 1074 & 9.64 & & & & \\
\hline 0506 & 06/07/2009 & 23.32 & 7.65 & 1174 & 7.54 & 0.36 & 10.27 & 0.08 & 0.29 \\
\hline 0506 & $07 / 09 / 2010$ & 21.09 & 7.70 & 1127 & 7.22 & & & & \\
\hline 0571 & $13 / 08 / 2008$ & 23.36 & 8.18 & 720 & 8.84 & 1.68 & 10.93 & 0.05 & 0.03 \\
\hline 0571 & $13 / 07 / 2009$ & 24.57 & 8.06 & 481 & 8.51 & $<0.06$ & 6.62 & $<0.05$ & 0.31 \\
\hline 0571 & $14 / 09 / 2010$ & 19.47 & 7.84 & 596 & 9.17 & & & & \\
\hline 0592 & $08 / 09 / 2010$ & 21.97 & 7.69 & 1024 & 6.60 & & & & \\
\hline 0647 & $28 / 06 / 2007$ & 17.34 & 7.80 & 2039 & 7.26 & $<0.01$ & 8.01 & 0.05 & 0.40 \\
\hline 0647 & 08/07/2009 & 19.67 & 7.68 & 2695 & 8.40 & $<0.06$ & 10.02 & $<0.05$ & 0.12 \\
\hline 0650 & $28 / 06 / 2007$ & 20.08 & 8.07 & 798 & 8.35 & 0.06 & 13.93 & 0.08 & 0.21 \\
\hline 1038 & $29 / 07 / 2010$ & 21.52 & 8.09 & 1195 & 8.69 & & & & \\
\hline 1047 & $18 / 08 / 2010$ & 24.86 & 8.24 & 287 & 8.35 & & & & \\
\hline 1156 & $19 / 08 / 2008$ & 21.76 & 7.86 & 751 & 7.14 & $<0.01$ & 12.26 & 0.05 & 0.52 \\
\hline 1156 & $15 / 07 / 2009$ & 25.32 & 8.36 & 492 & 9.45 & $<0.06$ & 6.38 & $<0.05$ & $<0.15$ \\
\hline 1156 & $14 / 09 / 2010$ & 20.68 & 8.08 & 610 & 10.40 & & & & \\
\hline 1167 & $15 / 07 / 2008$ & 26.60 & 8.06 & 787 & 6.70 & 0.05 & 9.04 & 0.24 & 0.05 \\
\hline 1295 & 02/07/2009 & 26.29 & 7.90 & 1990 & 8.10 & 0.15 & 18.92 & 0.32 & 0.26 \\
\hline 1304 & 03/08/2008 & 19.30 & 7.91 & 870 & 5.21 & $<0.01$ & 20.20 & 0.01 & 0.29 \\
\hline 1471 & $01 / 07 / 2010$ & 20.84 & 8.58 & 490 & 11.77 & & & & \\
\hline
\end{tabular}

Rino, 1992; Fott et al., 1965). John et al. (2002) consideran que no forma parte de la flora de algas natural del Reino Unido.

Las condiciones ambientales en las que se ha hallado T. javanicum en la Cuenca del Ebro son similares a las encontradas por Aboal et al. (1994) en la provincia de Alicante y por MarínMurcia \& Aboal (2007) en canales de riego de la provincia de Cáceres. En ambos casos la especie proliferaba en aguas cálidas de circulación lenta o estancada, bien oxigenadas, alcali- nas, de mineralización media, turbias y ricas en nutrientes. Sin embargo, las condiciones de bajo contenido en nutrientes y transparencia elevada del agua en los tramos medio y alto de los ríos Aragón y Matarraña, se asemejan a las condiciones ecológicas del material recolectado en Castilla-La Mancha (Moreno et al., 2011), Galicia (Penalta-Rodríguez \& López-Rodríguez, 2007) y podrían aproximarse más a las mencionadas por Calado \& Rino (1992) para Portugal, en sistemas de montaña con aguas más transpa- 
rentes. Todas las demás localidades, sin embargo, tienen en común el estar ubicadas en zonas de usos agrícola y ganadero. Esta aparente separación ambiental demandaría un estudio taxonómico más en profundidad.

Aunque se trata de una especie poco citada es probable que su distribución por el territorio español sea más amplia. Su desarrollo fundamentalmente estival y el carácter generalmente muy somero de los cuerpos de agua donde se alcanzan temperaturas relativamente elevadas, podría explicar la ampliación de su área de distribución a zonas más templadas.

Así como se ha hallado en otras regiones (Coute \& Tracanna, 1981; Aboal et al., 1994; Marín-Murcia \& Aboal, 2007), en la cuenca de Ebro muestra una gran afinidad por las aguas eutróficas y turbias, por lo que puede ser considerada indicadora de esas condiciones y debiera estudiarse su inclusión en la lista de especies indicadoras.

\section{AGRADECIMIENTOS}

Los autores están muy agradecidos a todas las personas que han colaborado en los diferentes muestreos que se han llevado a cabo en la Cuenca del Ebro en los últimos años. En especial a Andrés Mellado, Isidoro Pérez, José Miguel García, Pablo Jáimez-Cuéllar, Julio M. Luzón y José Antonio Palomino.

\section{BIBLIOGRAFÍA}

ABOAL, M., M. A. PUIG, A. SÁNCHEZ-GODÍNEZ \& G. SOLER. 1994. Algal standing-crop in some Mediterranean temporary rivers in southeastern Spain. Verhandlungen der Internationalen Vereinigung für Theoretische und Angewandte Limnologie 25: 1746-1750.

ABOAL, M., J. P. MARÍN, \& A. NIEVA. 2006. Tetrasporidium javanicum Möbius (Tetrasporales, Chlorophyta) ¿especie invasora? Resúmenes reunión científica de la Sociedad Española de Ficología. Algas (Boletín de la Sociedad Española de Ficología), 37: 4.

CALADO, A. J. \& J. A. RINO. 1992. Observations and taxonomic considerations on a Tetrasporidium (Chlorophyta, Tetrasporales) found in Portugal. Cryptogamie, Algologie, 13 (3): 157-167.

CHE. 2005. Metodología para el establecimiento del Estado Ecológico según la Directiva Marco del Agua. Protocolos de muestreo y análisis. Ministerio de Medio Ambiente. $47 \mathrm{pp}$.

CHE. 2007. Protocolo de desinfección de embarcaciones y equipos en masas de agua infectadas por mejillón cebra (Dreissena polymorpha). Ministerio de Medio Ambiente y Medio Rural y Marino. $16 \mathrm{pp}$.

COUTÉ, A. \& B. TRACANNA. 1981. Sur la présence en France de Tetrasporidium javanicum Möbius (Chlorophyta, Euchlorophyceae, Tetrasporales) et sa position systematique. Cryptogamie, Algologie, 2(3): 209-219.

DAY, S. A., R. P. WICKHAM, T. J. ENTWISTLE \& P. A. TYLER. 1995. Bibliographic check-list of non.marine algae in Australian. Flora of Australia Supplementary series 4: vii +276 pp. Australian Biological Resources Study. Canberra.

ENTWISLE, T. J. \& S. SKINNER. 2000. Non-marine algae of Australia: 4. Floristic survey of some colonial green macroalgae (Chlorophyta). Telopea, 9: 725-739.

ETTL, H. \& G. GÄRTNER. 1988. Chlorophyta II (Tetrasporales, Chlorococcales, Gloeodendrales). In: Ettl, H., Gerloff, J. \& Heynig, H. (eds). Süßwasserflora von Mitteleuropa. Vol. 10. Gustav Fischer Verlag, Jena. 436 pp.

FOTT, B., M. NOVÁKOVÁ \& T. KALINA. 1965. Morphology, reproduction and occurrence of a tropical alga, Tetrasporidium javanicum Möbius (Chlorophyceae). Preslia, 37: 380-386.

HU, H. \& Y. WEI. 2006. The freshwater algae of China. Systematics, taxonomy and ecology. Science Press. Pekin. 1023 pp.

ITC-MMA.EECC-1/06. 2006. ORDEN MAM/3207/ 2006, de 25 de septiembre, por la que se aprueba la instrucción técnica complementaria MMA-EECC$1 / 06$, determinaciones químicas y microbiológicas para el análisis de las aguas. BOE 250: 3632636440.

IYENGAR, M. O. P. 1932. Two little known genera of green algae (Tetrasporidium and Ecballocystis). Part I. Tetrasporidium javanicum Möbius. Annals of Botany 46: 191-199.

JOHN, D. M., B. A. WHITTON \& A. J. BROOK. (eds.) 2002. The Freshwater Algal Flora of the British 
Isles. Cambridge University Press. UK. 702 pp. LÓPEZ-RODRÍGUEZ, M. C. \& M. PENALTA-RODRÍGUEZ. 2007. Freshwater algae in Galician Central Macizo rivers (NW Spain) with new records for the Iberian Peninsula. Algological Studies 125: 57-77.

MARÍN-MURCIA, J. P. \& M. ABOAL. 2007. Estudio de las comunidades vegetales de los canales de Montijo y Lobón y de las condiciones que favorecen su desarrollo. (Informe Técnico). Confederación Hidrográfica del Guadiana. Ministerio de Medio Ambiente. 125 pp.

MORENO, J. L., R. ROS \& J. DE LAS HERAS. 2011. La flora acuática de los ríos y humedales de Castilla-La Mancha: comunidades, hábitats y medidas de conservación. En: Protección de la diversidad vegetal y de los recursos fitogenéticos en Castilla-La Mancha: la perspectiva ex situ y el compromiso del Jardín Botánico. E. Hernández \& J.M. Herranz (Coords.): 275-315. Instituto de Estudios Albacetenses. Albacete.

SARMA, Y., S. R. K. \& G. SURYANARAYANA. 1969. Observations on morphology, reproduction and cytology of Tetrasporidium javanicum Möebius from North India. Phycologia 8: 171-176.

SHERWOOD, A. R. 2004. New records of freshwater macroalgae and diatoms form Hawaian Islands. Bishop Museum Occasional Papers 79: 3-8. 